\title{
FLAT PRIMES AND THIN PRIMES
}

\author{
KEVIN A. BROUGHAN ${ }^{凶}$ and QIZHI ZHOU
}

(Received 18 December 2009)

\begin{abstract}
A number is called upper (lower) flat if its shift by $+1(-1)$ is a power of 2 times a squarefree number. If the squarefree number is 1 or a single odd prime then the original number is called upper (lower) thin. Upper flat numbers which are primes arise in the study of multi-perfect numbers. Here we show that the lower or upper flat primes have asymptotic density relative to that of the full set of primes given by twice Artin's constant, that more than $53 \%$ of the primes are both lower and upper flat, and that the series of reciprocals of the lower or the upper thin primes converges.
\end{abstract}

2000 Mathematics subject classification: primary 11A41; secondary 11B05, $11 \mathrm{~B} 83$.

Keywords and phrases: flat prime, thin prime, sieve.

\section{Introduction}

Some interesting subclasses of primes have been identified and actively considered. These include Mersenne primes (of the form $2^{p}-1$ ), Sophie Germain primes (of the form $2 p+1$ ), Fermat primes (of the form $2^{2^{n}}+1$ ), Cullen's primes (of the form $p 2^{p}+1$ ), Wieferich primes (which are primes $p$ such that $p^{2} \mid 2^{p-1}-1$ ), primes of the form $n^{2}+1$, of the form $n ! \pm 1$, and so on; see, for example, [10, Ch. 5] and the references therein. For any one of these classes, determining whether or not it is infinite has proved to be a very difficult problem.

In this paper we explore two classes of primes, the so-called lower or upper flat primes and the lower or upper thin primes. They have simple representations, and we are able to get an idea of their densities relative to the full set of primes.

These primes are similar to primes of the form $k \cdot 2^{e}+1$ considered by Erdős and Odlyzko, Chen and Sierpiński among others [3, 5, 12]. There the focus is mainly on the admissible values of odd integers $k$ with $k \leq x$, rather on the density of primes themselves having that structure. Erdős showed [5, Theorem 1] that the number $N(x)$ of odd numbers less than or equal to $x$ of the form $(p+1) / 2^{e}$ satisfies $c_{1} x \leq N(x) \leq c_{2} x$, where $c_{1}$ and $c_{2}$ are positive absolute constants. In the opposite direction, a simple modification of the derivation of Sierpiński [12] gives an infinite

(C) 2010 Australian Mathematical Publishing Association Inc. 0004-9727/2010 \$16.00 
number of integers $n$ (including an infinite set of primes) such that $n \cdot 2^{e}-1$ is composite for every $e=1,2,3, \ldots$.

DEFINITION 1. We say that a natural number $n$ is an upper flat number if $n+1=2^{e}$ or $n+1=2^{e} q_{1} \ldots q_{m}$ where $e \geq 1$ and the $q_{i}$ are distinct odd primes. If a prime $p$ is an upper flat number we say that $p$ is an upper flat prime. Let $F(x):=\#\{p \leq x$ : $p$ is an upper flat prime .

There are corresponding definitions of the terms lower flat number and lower flat prime obtained by replacing a shift by +1 with a shift by -1 .

DEFINITION 2. We say that a natural number $n$ is a lower flat number if $n-1=2^{e}$ or $n-1=2^{e} q_{1} \ldots q_{m}$ where $e \geq 1$ and the $q_{i}$ 's are distinct odd primes. If a prime $p$ is a lower flat number we say that $p$ is a lower flat prime.

It is straightforward to show that the density of upper flat or lower flat numbers is the same as that of the odd squarefree numbers, that is, the number up to $x$ is given by $4 x / \pi^{2}+O(\sqrt{x})[13]$.

DEFINITION 3. We say that a natural number $n$ is an upper thin number if $n+$ $1=2^{e} q$ or $n+1=2^{e}$ where $e \geq 1$ and $q$ is an odd prime. If a prime $p$ is an upper thin number we say that $p$ is an upper thin prime. Let $T(x):=\#\{p \leq x$ : $p$ is an upper thin prime\}.

DEFINITION 4. We say that a natural number $n$ is a lower thin number if $n-1=$ $2^{e} q$ or $n-1=2^{e}$ where $e \geq 1$ and $q$ is an odd prime. If a prime $p$ is a lower thin number we say that $p$ is a lower thin prime.

For example, among the first 100 primes, 75 primes are upper flat and among the first 1000 primes, 742 are upper flat. For upper thin primes the corresponding numbers are 38 and 213, respectively. The first 10 upper flat primes are 3, 5, 7, 11, 13, 19, 23, 29, 31, and 37. The first 10 upper thin primes are 3, 5, 7, 11, 13, 19, 23, 31, 37 and 43 .

If $M(x)$ is the number of Mersenne primes up to $x$, then clearly, for all $x \geq 1$, $M(x) \leq T(x) \leq F(x) \leq \pi(x)$, where for each $x>0, \pi(x)$ is the number of primes up to $x$.

These types of number arise frequently in the context of multi-perfect numbers, that is, numbers that satisfy $\sigma(n)=k n,(k \geq 2)$, where $\sigma(n)$ is the sum of the positive divisors of $n$. For example, when $k=3$ all of the known examples of so-called 3-perfect numbers are

$$
\begin{aligned}
& c_{1}=2^{3} \cdot 3 \cdot 5, \\
& c_{2}=2^{5} \cdot 3 \cdot 7, \\
& c_{3}=2^{9} \cdot 3 \cdot 11 \cdot 31, \\
& c_{4}=2^{8} \cdot 5 \cdot 7 \cdot 19 \cdot 37 \cdot 73, \\
& c_{5}=2^{13} \cdot 3 \cdot 11 \cdot 43 \cdot 127, \\
& c_{6}=2^{14} \cdot 5 \cdot 7 \cdot 19 \cdot 31 \cdot 151 .
\end{aligned}
$$


Each $c_{i}-1$ is an upper flat number and each odd prime appearing on the right-hand side is upper thin.

This paper is organized as follows. In Section 2 we first show that the asymptotic density of upper thin numbers up to $x$, as $x \rightarrow \infty$, is equal to that of the primes up to $x$. In Section 3 we show that the density of upper flat primes up to $x$, relative to the density of all primes, is given by $2 A$ where $A$ is Artin's constant. A corollary to this is that there is an upper flat prime in every interval $[x,(1+\epsilon) x]$, for any $\epsilon>0$ and sufficiently large $x$. This is followed by a demonstration that primes which are both lower and upper flat have an asymptotic density and constitute more than half of all primes. In Section 4 we then show that the upper thin primes are sufficiently sparse that the sum of their reciprocals converges.

We use Landau's $O, o$, and $\ll$ notation. The symbols $p, q$ are restricted to be rational primes.

\section{Upper or lower thin numbers}

THEOREM 5. As $x \rightarrow \infty$, the asymptotic density of upper or lower thin numbers up to $x$ is the same as that of the primes up to $x$.

PROOF. Firstly, the number of upper or lower thin numbers up to $x, N(x)$, is given by

$$
N(x)=\sum_{n=1}^{\lfloor\log x / \log 2\rfloor} \pi\left(\frac{x}{2^{n}}\right) .
$$

Next we will show that $\lim _{x \rightarrow \infty} N(x) / \pi(x)=1$. To this end first consider a single term in the sum. By [11], there is a positive real absolute constant $\alpha$ such that for $x$ sufficiently large,

$$
\frac{x}{\log x+\alpha}<\pi(x)<\frac{x}{\log x-\alpha} .
$$

Therefore, for all $n \in \mathbb{N}$ such that $1 \leq n \leq\lfloor\log x / \log 2\rfloor$ (which makes the numerators and denominators of the ratios below positive for sufficiently large $x$ ),

$$
l b:=\frac{1-\frac{\alpha}{\log x}}{1+\frac{\alpha}{\log x}-\frac{n \log 2}{\log x}}<\frac{2^{n} \pi\left(\frac{x}{2^{n}}\right)}{\pi(x)}<\frac{1+\frac{\alpha}{\log x}}{1-\frac{\alpha}{\log x}-\frac{n \log 2}{\log x}}=: u b .
$$

Clearly $l b$ and $u b$ tend to 1 as $x \rightarrow \infty$ uniformly for $n$ in the range $1 \leq n \leq$ $\log x / \log \log x=: b$. The difference between the upper and lower bounds is

$$
u b-l b=\frac{1}{d}\left(\frac{4 \alpha}{\log x}-\frac{2 \alpha n \log 2}{\log ^{2} x}\right) \leq \frac{4 \alpha}{d \log x},
$$

where

$$
\begin{aligned}
d & =\left(1-\frac{\alpha}{\log x}-\frac{n \log 2}{\log x}\right)\left(1+\frac{\alpha}{\log x}-\frac{n \log 2}{\log x}\right) \\
& =1-\frac{\alpha^{2}}{\log ^{2} x}+\frac{n^{2} \log ^{2} 2}{\log ^{2} x}-\frac{2 n \log 2}{\log x} \geq \frac{1}{4},
\end{aligned}
$$


so

$$
u b-l b \leq \frac{16 \alpha}{\log x},
$$

for all $n$ in the given range and $x$ sufficiently large.

Now ensure that $x$ is sufficiently large so that $\log 2 / \log \log x<0.5$ and

$$
\begin{aligned}
|1-l b| & \leq \frac{\frac{2 \alpha}{\log x}-\frac{n \log 2}{\log x}}{1+\frac{\alpha}{\log x}-\frac{n \log 2}{\log x}} \\
& \leq \frac{\frac{2 \alpha}{\log x}}{\frac{1}{2}+\frac{\alpha}{\log x}} \\
& \leq \frac{4 \alpha}{\log x} .
\end{aligned}
$$

This implies, for $x$ sufficiently large and $1 \leq n \leq b$, that

$$
\begin{aligned}
\left|\frac{2^{n} \pi\left(\frac{x}{2^{n}}\right)}{\pi(x)}-1\right| & \leq\left|\frac{2^{n} \pi\left(\frac{x}{2^{n}}\right)}{\pi(x)}-l b\right|+|l b-1| \\
& \leq|u b-l b|+|l b-1|<\frac{20 \alpha}{\log x} .
\end{aligned}
$$

Using this bound we derive

$$
\begin{aligned}
\left|\sum_{n \leq b} \frac{\pi\left(\frac{x}{2^{n}}\right)}{\pi(x)}-1\right| & \leq\left|\frac{\sum_{n \leq b} \pi\left(\frac{x}{2^{n}}\right)}{\pi(x)}-\frac{\sum_{n \leq b} \frac{\pi(x)}{2^{n}}}{\pi(x)}\right|+\sum_{n>b} \frac{1}{2^{n}} \\
& \leq \sum_{n \leq b} \frac{1}{2^{n}}\left|\frac{2^{n} \pi\left(\frac{x}{2^{n}}\right)}{\pi(x)}-1\right|+o(1) \\
& \leq \sum_{1 \leq n} \frac{20 \alpha}{2^{n} \log x}+o(1)=o(1)
\end{aligned}
$$

as $x \rightarrow \infty$.

For the remaining part of the summation range for $N(x)$, namely for $b<n \leq$ $\lfloor\log x / \log 2\rfloor$, note that this corresponds to values of $x$ and $n$ which satisfy

$$
\frac{x}{2^{n}} \leq x^{1-\log 2 / \log \log x} .
$$

Using $\pi(x) \leq x$ and defining

$$
S(x):=\sum_{n=b}^{\lfloor\log x / \log 2\rfloor} \pi\left(\frac{x}{2^{n}}\right) \ll \log x \cdot x^{1-\log 2 / \log \log x},
$$

it follows (using, say, l'Hôpital's rule) that $S(x) / \pi(x) \rightarrow 0$ as $x \rightarrow \infty$. Hence $N(x) / \pi(x) \rightarrow 1$. 
From this we have consequences such as that an infinite number of successive primes are separated by a thin number and vice versa.

\section{Upper or lower flat primes}

Define the so-called logarithmic integral for $x \geq 2$ :

$$
\operatorname{Li}(x):=\int_{2}^{x} \frac{d t}{\log t} .
$$

THEOREM 6. For all $H>0$,

$$
F(x)=2 \prod_{p}\left(1-\frac{1}{p(p-1)}\right) \operatorname{Li}(x)+O\left(\frac{x}{\log ^{H} x}\right),
$$

that is, the relative density of upper or lower flat primes is $2 A=0.7480 \ldots$ where $A$ is Artin's constant.

ProOF. Fix $e \geq 1$ and let $x$ and $y$ satisfy $1<y<x$ and be sufficiently large. Let $H>0$ be the given positive constant. Define

$$
F_{e}(x):=\#\left\{p \leq x: p \text { is prime and } m \text { squarefree such that } 2^{e} m=p+1\right\} .
$$

If $\mu(n)$ is the Möbius function and $\mu_{2}(n)$ the characteristic function of the squarefree numbers, we can write

$$
\mu_{2}(n)=\sum_{d^{2} \mid n} \mu(d)
$$

Then

$$
\begin{aligned}
F_{e}(x) & =\sum_{\substack{p \leq x \\
p+1=2^{e} m}} \mu_{2}(m)=\sum_{p \leq x} \sum_{\substack{a: a \geq 1 \\
a^{2} b 2^{e}=p+1}} \mu(a) \\
& =\Sigma_{1}+\Sigma_{2},
\end{aligned}
$$

where

$$
\Sigma_{1}:=\sum_{p \leq x} \sum_{\substack{a: 1 \leq a \leq y \\ a^{2} b 2^{e}=p+1}} \mu(a) \text { and } \Sigma_{2}:=\sum_{p \leq x} \sum_{\substack{a>y \\ a^{2} b 2^{e}=p+1}} \mu(a) .
$$

Now using the Bombieri-Vinogradov theorem [4, Section 28] for the number of primes in an arithmetic progression, which is valid with a uniform error bound for the values of $e$ which will be needed,

$$
\begin{aligned}
\Sigma_{1} & =\sum_{a \leq y} \mu(a) \sum_{\substack{p: p \leq x \\
p \equiv-1 \bmod 2^{e} a^{2}}} 1 \\
& =\sum_{a \leq y} \mu(a)\left(\frac{\operatorname{Li}(x)}{\phi\left(2^{e} a^{2}\right)}+O\left(\frac{x}{\log ^{2 H+1} x}\right)\right) \\
& =\left(\sum_{a \geq 1} \frac{\mu(a)}{\phi\left(2^{e} a^{2}\right)}\right) \operatorname{Li}(x)+O\left(\frac{x}{\log x} \sum_{a>y} \frac{1}{\phi\left(2^{e} a^{2}\right)}\right)+O\left(\frac{x y}{\log ^{2 H+1} x}\right) .
\end{aligned}
$$


Note that the function $g(n):=2^{1-e} \phi\left(2^{e} n^{2}\right)$ is multiplicative, and the series in the sum below is absolutely convergent, so the coefficient of $\operatorname{Li}(x)$ may be rewritten

$$
\begin{aligned}
\frac{1}{2^{e-1}} \sum_{a \geq 1} \frac{2^{e-1} \mu(a)}{\phi\left(2^{e} a^{2}\right)} & =\frac{1}{2^{e-1}} \prod_{p}\left(1-\frac{2^{e-1}}{\phi\left(2^{e} p^{2}\right)}\right) \\
& =\frac{1}{2^{e-1}} \frac{3}{4} \prod_{p \text { odd }}\left(1-\frac{1}{p^{2}-p}\right)=\frac{3 A}{2^{e}} .
\end{aligned}
$$

Now consider the sum in the first error term for $\Sigma_{1}$ :

$$
\sum_{a>y} \frac{1}{\phi\left(2^{e} a^{2}\right)} \ll \sum_{a>y} \frac{1}{2^{e} \phi\left(a^{2}\right)} \ll \frac{1}{2^{e}} \sum_{a>y} \frac{\log \log a}{a^{2}} .
$$

Therefore

$$
O\left(\frac{x}{\log x} \sum_{a>y} \frac{1}{\phi\left(2^{e} a^{2}\right)}\right)=O\left(\frac{x \log \log y}{2^{e} y \log x}\right) .
$$

For the second sum,

$$
\left|\Sigma_{2}\right| \leq \sum_{p<x} \sum_{\substack{a>y \\ p+1=2^{e} a^{2} b}} 1 \leq \sum_{\substack{a>y \\ 2^{e} a^{2} b \leq x}} 1=O\left(\frac{x}{2^{e} y}\right),
$$

and therefore

$$
F_{e}(x)=\frac{3 A}{2^{e}} \operatorname{Li}(x)+O\left(\frac{x \log \log y}{2^{e} y \log x}\right)+O\left(\frac{x}{2^{e} y}\right)+O\left(\frac{x y}{\log ^{2 H+1} x}\right) .
$$

If we choose $y=\log ^{H} x$, then

$$
F_{e}(x)=\frac{3 A}{2^{e}} \operatorname{Li}(x)+O\left(\frac{x}{\log ^{H+1} x}\right) .
$$

Now let

$$
D_{e}(x):=\#\left\{p \leq x: p \text { is prime, } p+1=2^{e} m \text {, with } m \text { squarefree and odd }\right\} .
$$

By [8, Theorem 2],

$$
D_{1}(x)=A \cdot \operatorname{Li}(x)+O\left(x / \log ^{H+1} x\right) .
$$

Considering the even and odd cases, for all $e \geq 1$, we have $F_{e}(x)=D_{e}(x)+D_{e+1}(x)$ so

$$
F_{1}(x)+F_{2}(x)+\cdots=D_{1}(x)+2\left(D_{2}(x)+D_{3}(x)+\cdots\right)
$$


and therefore

$$
\begin{aligned}
F(x) & =\sum_{e=1}^{\lfloor\log x / \log 2\rfloor} D_{e}(x)+O(\log x) \\
& =\frac{1}{2}\left(D_{1}(x)+F_{1}(x)+F_{2}(x)+\cdots\right)+O(\log x) \\
& =\frac{A}{2}\left(1+\frac{3}{2^{1}}+\frac{3}{2^{2}}+\cdots\right) \operatorname{Li}(x)+O\left(\frac{x}{\log ^{H+1} x}\right) \\
& =2 A \operatorname{Li}(x)+O\left(\frac{x}{\log ^{H} x}\right)
\end{aligned}
$$

and this completes the proof for upper flat primes. The proof for lower flat primes is similar.

Since $2 A>0.74$, the relative density of either lower flat or upper flat primes is greater than $74 \%$. Thus, in the worst possible case the density of primes which are neither lower nor upper flat would be less than $26 \%+26 \%=52 \%$, leading to a lower bound of $48 \%$ for the density of the set of primes which are both upper flat and lower flat. However, this figure underestimates the proportion of such primes-see Theorem 8 and its corollary below.

COROLlary 7. For all $\epsilon>0$ and $x \geq x_{\epsilon}$ there exist an upper flat prime and a lower flat prime in the interval $[x,(1+\epsilon) x]$.

Proof. Since $F(x)=2 A x / \log x+O\left(x / \log ^{2} x\right)$, for fixed $\epsilon>0$ we have

$$
F(x+\epsilon x)-F(x)=\frac{2 A \epsilon x}{\log x}+O\left(\frac{x}{\log ^{2} x}\right),
$$

which is strictly positive for all $x$ sufficiently large.

Note also that it would be possible to adapt the method of Adleman et al. [1, Proposition 9] to count lower or upper flat primes in arithmetic progressions.

THEOREM 8. Let the constant $H>0$ and the real variable $x$ be sufficiently large. Let the set of primes which are both lower and upper flat and which are less than $x$ be given by

$$
\begin{gathered}
B(x)=\left\{p \leq x: \exists e \geq 1, f \geq 1 \text { and odd squarefree } u, v \text { so } p-1=2^{e} v,\right. \\
\left.p+1=2^{f} u\right\} .
\end{gathered}
$$

Then

where

$$
B(x)=A_{2} \operatorname{Li}(x)+O\left(\frac{x}{\log ^{H} x}\right)
$$

$$
A_{2}=\prod_{p \text { odd }}\left(1-\frac{2}{p^{2}-p}\right)=0.53511 \ldots
$$


PROOF. Let $e, f \geq 1$ and define the sets

$$
\begin{aligned}
L_{e} & :=\left\{p \leq x: \exists \text { odd squarefree } v \text { so } p-1=2^{e} v\right\}, \\
U_{f} & :=\left\{p \leq x: \exists \text { odd squarefree } u \text { so } p+1=2^{f} u\right\} .
\end{aligned}
$$

Then $L_{1} \cap U_{1}=\emptyset$ and $L_{e} \cap U_{f}=\emptyset$ for all $e \geq 2, f \geq 2$, so we can write

$$
B(x)=\left\{\bigcup_{f \geq 2} L_{1} \cap U_{f}\right\} \cup\left\{\bigcup_{e \geq 2} U_{1} \cap L_{e}\right\}
$$

where all of the unions are disjoint.

Now fix $e \geq 2$. We will first estimate the size of $U_{1} \cap L_{e}$, where

$$
U_{1} \cap L_{e}=\left\{p \leq x: \exists \text { odd squarefree } u, v \text { so } p+1=2 u, p-1=2^{e} v\right\} .
$$

Then

$$
\begin{aligned}
& \# U_{1} \cap L_{e}=\sum_{p \leq x} \sum_{\begin{array}{c}
p+1=2 u, \\
p-1=2^{e} v, \\
u, v \text { odd and squarefree }
\end{array}} 1 \\
& =\sum_{p \leq x} \sum_{a, b \text { odd, }(a, b)=1,} \mu(a) \mu(b) \\
& p \equiv-1 \bmod a^{2}, a^{2} \leq x / 2 \\
& p \equiv 1 \bmod b^{2}, b^{2} \leq x / 2^{e} \\
& p \equiv 1+2^{e} \bmod 2^{e+1} \\
& =\sum_{p \leq x} \sum_{a, b \text { odd },(a, b)=1, a^{2} b^{2} \leq x^{2} 2^{-e-1},} \mu(a) \mu(b) \\
& p \equiv u \bmod 2^{e+1} a^{2} b^{2} \\
& =\sum_{p \leq x} \sum_{\substack{d \text { odd }, d^{2} \leq x^{2} 2^{-e-1}, p \equiv u \bmod 2^{e+1} d^{2}}} \tau^{*}(d) \mu(d)
\end{aligned}
$$

where $u$, the residue obtained through an application of the Chinese remainder algorithm, is dependent on $d$ and $e$, and $\tau^{*}(d)$ is the number of unitary divisors of $d$, a multiplicative function with $\tau^{*}(p)=2$. This function arises because for fixed $d \geq 1$, the number of decompositions $d=a b$ with $(a, b)=1$ is $\tau^{*}(d)$.

We then split and reverse the sum in a similar manner to the proof of Theorem 6 to arrive at

$$
\begin{aligned}
\# U_{1} \cap L_{e} & =\left(\sum_{d \geq 1, d \text { odd }} \frac{\tau^{*}(d) \mu(d)}{\phi\left(2^{e+1} d^{2}\right)}\right) \operatorname{Li}(x)+O\left(\frac{x}{\log ^{H+1} x}\right) \\
& =\frac{1}{2^{e}} \prod_{p \text { odd }}\left(1-\frac{2}{p^{2}-p}\right) \operatorname{Li}(x)+O\left(\frac{x}{\log ^{H+1} x}\right) .
\end{aligned}
$$

Summing over $e \geq 2$ and, noticing that the sizes for each corresponding $L_{1} \cap U_{e}$ are the same, we obtain the stated value of $B(x)$. 


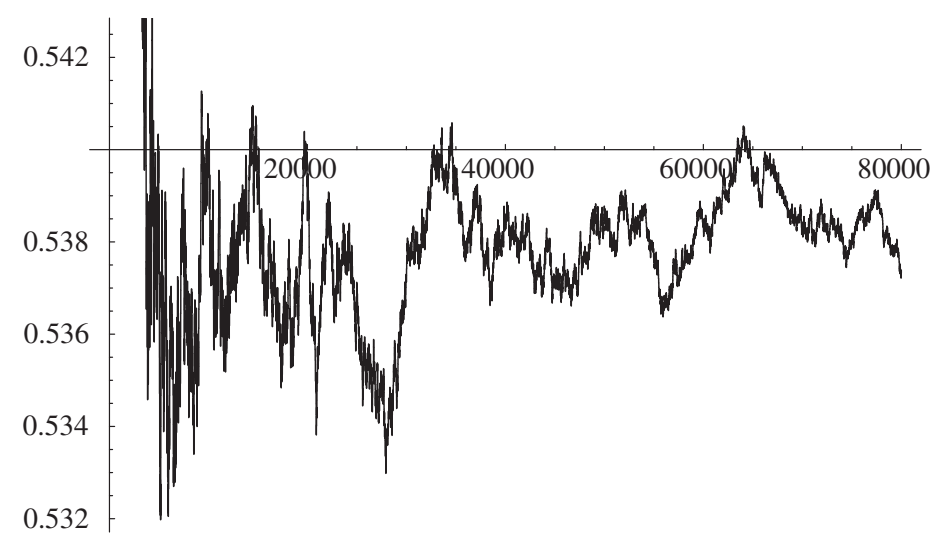

FIGURE 1 . The ratio $B(x) / \pi(x)$ for $1 \leq x \leq 8 \cdot 10^{4}$.

Figure 1 compares the number of primes up to 80000 with the number of primes up to 80000 which are both lower and upper flat.

COROLLARY 9. It follows from Theorems 6 and 8 that the set of rational primes may be divided into four disjoint classes: those both lower and upper flat (about 54\%), those either lower or upper flat but not both (each about 21\%), and those neither upper nor lower flat (4\%).

COROLlary 10. It follows that primes which are both upper and lower flat and congruent to 1 modulo 4 (3 modulo 4 ) are a positive relative proportion of all primes. These must therefore be of the form $p=2 s-1(p=2 s+1)$ where $s$ is odd and squarefree.

Comment. Note that not both $p+1$ and $p-1$ can be squarefree for odd primes $p$, and that the same applies to $p \pm h$ for odd shifts $h$. It appears, numerically, that for fixed odd $h$, the proportion of primes $p$ with both $p+h$ and $p-h$ flat is always over $50 \%$, with smaller proportions for $h \equiv \pm 1 \bmod 6$ and larger for $h \equiv 3 \bmod 6$. The larger proportions appear to be all significantly larger than the smaller. This warrants further investigation.

\section{Upper and lower thin primes}

Using the method of Chen, Heath-Brown [7, Lemma 1] showed that if $H(x)$ is the number of primes such that $p \leq x$ and either $p+1=2 p_{1}$ or $p+1=2 p_{1} p_{2}$, with the $p_{i}$ being odd primes, then $H(x) \gg x / \log ^{2} x$. Based on this evidence, the HardyLittlewood-Bateman-Horn conjecture [2,6], and numerical evidence, we are led to the following conjecture.

COnjecture. The number of upper thin primes up to $x$ satisfies $T(x) \gg x / \log ^{2} x$ and has the same asymptotic density as the number of twin primes up to $x$, and the same is true for the lower thin primes. 
The order of difficulty of this conjecture appears to be similar to showing that there are an infinite number of twin primes or Sophie-Germain primes. As usual, upper bounds are much easier to work with and we obtain the following theorem.

Theorem 11. As $x \rightarrow \infty, T(x) \ll x / \log ^{2} x$.

PROOF. First let $e \geq 1$ be fixed and apply the sieve of Brun in the same manner as for the classical twin primes problem (for example, [14, Theorem 4]) to count $J_{e}(x):=\#\left\{p \leq x: 2^{e} p-1\right.$ is prime $\}$. Note that if $\mathcal{A}=\left\{m\left(2^{e} m-1\right): m \leq x\right\}$ and $\rho(d)$ is the number of solutions modulo $d$ which satisfy $m\left(2^{e} m-1\right) \equiv 0 \bmod$ $d$, then $\rho$ is a multiplicative function. Also $\rho(2)=1$ and $\rho(p)=2$ for odd primes $p$, leading to the same bound as in the twin primes problem, namely $J_{e}(x) \ll x / \log ^{2} x$.

Now we use the fact that, for all $m \geq 1$,

$$
\sum_{n=1}^{m} \frac{2^{n}}{n^{2}}<5 \frac{2^{m}}{m^{2}} .
$$

Finally, let $x$ be large and choose $m \in \mathbb{N}$ so that $2^{m} \leq x<2^{m+1}$. Then

$$
\begin{aligned}
T(x) & =\sum_{e=1}^{\lfloor\log x / \log 2\rfloor}\left(J_{e}\left(\frac{x}{2^{e}}\right)+O(1)\right) \\
& \ll \sum_{e=1}^{\lfloor\log x / \log 2\rfloor-1} \frac{x}{2^{e}} \frac{1}{\log ^{2} \frac{x}{2^{e}}}+O(\log x) \\
& \leq \sum_{e=0}^{\left\lfloor\log 2^{m+1} / \log 2\right\rfloor-1} \frac{2^{m+1}}{2^{e}} \frac{1}{\log ^{2} \frac{2^{m+1}}{2^{e}}}+O(\log x) \\
& =\frac{1}{\log ^{2} 2} \sum_{n=1}^{m+1} \frac{2^{n}}{n^{2}}+O(\log x) \\
& <5 \frac{1}{\log ^{2} 2} \frac{2^{m+1}}{(m+1)^{2}}+O(\log x) \quad \text { by }(1) \\
& \ll \frac{x}{\log ^{2} x},
\end{aligned}
$$

completing the proof of the theorem.

So the asymptotic bound is the same as that for twin primes. In the same manner as originally derived by Brun for the sum of reciprocals of the twin primes (for example, [9, Theorem 6.12]) we obtain the following corollary.

COROLLARY 12. The sum of the reciprocals of the upper or lower thin primes is finite. 


\section{Acknowledgements}

We are grateful for the suggestions of a helpful referee, and to Igor Shparlinski for making us aware of the theorem of Heath-Brown. The computations and graphics were produced using Mathematica.

\section{References}

[1] L. M. Adleman, C. Pomerance and R. S. Rumely, 'On distinguishing prime numbers from composite numbers', Ann. of Math. (1) 117 (1983), 173-206.

[2] P. T. Bateman and H. Diamond, Analytic Number Theory: An Introductory Course (World Scientific, Singapore, 2004).

[3] Y. G. Chen, 'On integers of the form $k \cdot 2^{n}+1$ ', Proc. Amer. Math. Soc. 129 (2000), 355-361.

[4] H. Davenport, Multiplicative Number Theory, 3rd edn (Springer, New York, 2000).

[5] P. Erdôs and A. M. Odlyzko, 'On the density of odd integers of the form $(p-1) 2^{-n}$ and related questions', J. Number Theory 11 (1979), 257-263.

[6] G. H. Hardy and J. E. Littlewood, 'Some problems of "Partitio numerorum"; III: On the expression of a number as a sum of primes', Acta Math. 44 (1923), 1-70.

[7] D. R. Heath-Brown, 'Artin's conjecture for primitive roots', Q. J. Math. Oxford Ser. (2) 37 (1986), 27-38.

[8] L. Mirsky, 'The number of representations of an integer as the sum of a prime and a $k$-free integer', Amer. Math. Monthly 56 (1949), 17-19.

[9] M. B. Nathanson, Additive Number Theory: The Classical Bases (Springer, New York, 1996).

[10] P. Ribenboim, The New Book of Prime Number Records, 3rd edn (Springer, New York, 1996).

[11] J. B. Rosser and L. Schoenfeld, 'Approximate formulas for some functions of prime numbers', Illinois J. Math. 6 (1962), 64-94.

[12] W. Sierpiński, 'Sur un problème concernant les nombres $k \cdot 2^{n}+1$ ', Elem. Math. 15 (1960), 73-74.

[13] D. Suryanarayana, 'The number of $k$-free integers $\leq x$ which are coprime to $n$ ', Indian J. Math. 11 (1969), 131-139.

[14] G. Tenenbaum, Introduction to Analytic and Probabilistic Number Theory (Cambridge University Press, Cambridge, 1995).

\section{KEVIN A. BROUGHAN, University of Waikato, Hamilton, New Zealand e-mail: kab@waikato.ac.nz}

QIZHI ZHOU, University of Waikato, Hamilton, New Zealand

e-mail: qz49@waikato.ac.nz 